\title{
Simultaneous Measurements for Tunable Laser Source Linewidth with Homodyne Detection
}

\author{
Adnan H. Ali \\ Technical college / Baghdad- Iraq \\ Tel: 96-4-770-794-8995_E-mail: Adnan_h_ali@yahoo.com
}

Received: April 13, 2011

Accepted: June 17, 2011

doi:10.5539/cis.v4n4p138

\begin{abstract}
The linewidth characteristics of a commercial tunable laser source (TLS) are measured experimentally employing self-homodyne detection method. Three linewidths are obtained at different operating wavelengths with two optical fiber delay lengths. It is found that a slightly higher values are obtained with a longer length of the delay fiber. The long of $(50 \mathrm{~km}$ and $25 \mathrm{~km})$ of single mode fiber SMF is enough to provide delay for the self-homodyne linewidth measurement.
\end{abstract}

Keywords: Linewidth, Tunable laser source (TLS), Homodyne detection

\section{Introduction}

Recent advances in laser technology have made it possible to fabricate diode lasers or fiber lasers with emission linewidths less than $1 \mathrm{MHz}$ in external cavity configurations; laser linewidths of the order of kilohertz have been reported. Many applications require laser sources that offer a narrow linewidth, a high degree of coherence and low optical phase noise. The need to measure and characterize the narrow linewidth of these laser sources often arises in such applications as coherent optical communication, optical precision metrology, and high-resolution spectroscopy[Ludvigsen, Hanne et al. 1998][ X. Chen 2006][ Gary L. Wood 2006] .

The developed laser sources show improved coherence properties and linewidth in the sub-megahertz range will be available in a near future even in monolithic semiconductor lasers. The linewidth measurement of such lasers is a new challenge, since classical self-homodyne or self-heterodyne techniques require excessive lengths of delaying fibre and are thus not practicable[Alain Kueng, Luc Thevenaz, Philippe A. Robert, 1996]. Typical linewidths of stable free-running single-frequency solid-state lasers are a few kilohertz range, whereas the linewidths of semiconductor lasers are often in the megahertz range. Much smaller linewidths, sometimes even below $1 \mathrm{~Hz}$, can be reached by stabilization of lasers, which can be achieved using ultrastable reference cavity[Smith, S. P. et al.,1991]. The linewidth of a single frequency laser is the full width at half maximum (FWHM) of the optical spectrum. For simple cases, the laser linewidth only arises from quantum fluctuations. More precisely, it is the width of the power spectral density of the emitted electric field in terms of frequency, wavenumber or wavelength. The linewidth is strongly related to the temporal coherence, characterized by the coherence time or coherence length[K. M. Abramski, et al., 2005].

The basic idea of homodyne technique is to convert the optical phase or frequency fluctuations of the laser into variations of light intensity in a Mach-Zehnder type interferometer. In the interferometer, the optical field is mixed with a delayed replica of itself and the interference signal is detected with a fast photodiode [R. Paschotta. 2010]. The laser linewidth is then deduced from the recorded spectrum. Self-homodyne linewidth measurement is simpler than the coherent detection because it does not require a tunable local oscillator; however, it has a few disadvantages. First, in order for the interferometer to operate in the incoherent regime, the length difference between the two arms has to be large enough. This requirement may be difficult to satisfy when the linewidth of the light source is very narrow. To ensure an accurate measurement of this linewidth, the length of the delay line in one of the two interferometer arms has to be much longer than $10 \mathrm{~km}$. Another problem of self-homodyne measurement is that the central RF frequency is at zero, while most RF spectrum analyzers have high noise levels in this very low-frequency region.

The tunable laser source TLS has a high output power with high side mode suppression ratio and can accurately set wavelength range to accommodate various aspects of dense wavelength division multiplexing (DWDM) use. These characteristics are excellent for evaluating optical fiber amplifiers and components, DWDM systems, etc. 
The gain medium of the TLS is a conventional laser diode but the internal Fabry Perot resonator is disabled by an antireflection coating on one of the facets. An external cavity is created by adding an external diffraction grating which acts both as a mirror and a wavelength-selective element. The output power, single-mode operation and wavelength stability strongly depend on temperature stability[Ch. Spiegelberg, et al. 2004].

The TLS is a compact manual tunable laser for sensing, test and measurement, and telecommunication applications. It can provide more than $70 \mathrm{~mW}$ of optical output power with continuous tuning wavelength. It has a tuning range up to $20 \mathrm{~nm}$ within $1530-1620 \mathrm{~nm} \& 1030-1080 \mathrm{~nm}$. This tunable laser source also has a narrow linewidth of less than $0.02 \mathrm{~nm}$, which is necessary for many applications in telecommunication, sensor, biomedical and instrumentation et al[Geng J. et al. 2006].

Single frequency fiber lasers can achieve linewidths of a few kilohertz, or sometimes even below $1 \mathrm{kHz}$. With serious efforts at active stabilization, sub-hertz linewidths are sometimes achieved. The linewidth of a laser diode is typically in the megahertz region, but it can also be reduced to a few kilohertz, e.g. in external-cavity diode lasers, particularly with optical feedback from a high-finesse reference cavity.

A laser linewidth can be measured with a variety of techniques such as using optical spectral analysis as well as converting the frequency fluctuation into intensity fluctuation[K. M. Abramski, et al., 2005]. For large linewidths of more than $10 \mathrm{GHz}$, the first technique of optical spectrum analysis using diffraction grating is normally used. The latter technique uses frequency discriminator such as an unbalanced interferometer or a high-finesse reference cavity as the converter. For single-frequency lasers, the self-heterodyne technique is often used, which involves recording a beat note between the laser output and a frequency-shifted and delayed version of it. For sub-kilohertz linewidths, the ordinary self-heterodyne technique usually becomes impractical, but it can be extended by using a recirculating fiber loop with an internal fiber amplifier. Very high resolution can also be obtained by recording a beat note between two independent lasers, where either the reference laser has significantly lower noise than the device under test, or both lasers have similar performance.

\section{Self-homodyne detection}

In coherent detection, the frequency of the input optical signal is down converted to the RF domain through mixing with a local oscillator. Self-homodyne detection eliminates the requirement of a local oscillator, and the optical signal mixes with a delayed version of itself. Fig. 1 shows the self-homodyne detection set-up, where two delay lines are used between two directional couplers to form a Mach-Zehnder configuration [R. Paschotta . 2010].

The fiber delay length in one arm of the set-up is $25 \mathrm{~km}$ corresponding to a time delay $\tau$ of $125 \mu$ s and system resolution of $(\mathrm{c} / \mathrm{nL}) / 2$ where $\mathrm{c}$ is the speed of light $\mathrm{n}$ is the refractive index of the fiber and $\mathrm{L}$ is the fiber length.

As shown in Fig.1, the input signal optical field can be expressed as;

$$
E(t)=\sqrt{P \exp [j(w t+\phi(t))]}
$$

Where $\mathrm{P}$ is the power of the optical signal, $w t$ is the frequency and $\phi(t)$ is the phase of the signal. The optical

field is divided into two paths by the Mach-zehnder interferometer, where one side of the path consists of a long length of delay fiber. The composite optical field at output end of the interferometer is given as:

$$
E T(t)=A 1 \exp \left[j\left(w\left(t-\tau_{1}\right)+\phi\left(t-\tau_{1}\right)\right)\right]+A 2 \exp \left[j\left(w\left(t-\tau_{2}\right)+\phi\left(t-\tau_{2}\right)\right)\right]
$$

where $\tau_{1}$ and $\tau_{2}$ are the propagation delays of the two interferometer arms and A1 and A2 are the amplitudes of the fields emerging from these two arms. The output light is measured by a photo-detector, which convert the optical power to a current. The photocurrent can be represented by:

$$
I(t)=\mathscr{R}\{P 1+P 2+2 \sqrt{P 1 P 2} \cos [\omega \Delta \tau+\Delta \emptyset(t)]\}
$$

Where P1 (t) and P2 (t) are the powers of the optical signals passing through the two arms, $\Delta \tau=\Delta \tau_{\mathbf{2}}-\Delta \tau_{1}$ is their differential time delay, and $\Delta \phi(t)=\phi(t-\tau 2)-\phi(t-\tau 1)$ is their differential phase. Since the phase noise has a Gaussian statistics, in a stationary process we have 


$$
\Delta \phi(t)=\phi(t)-\phi(t-\Delta \tau)
$$

In a self-homodyne detection system, differential delay $\Delta$ is an important parameter. If the differential delay is much longer than the coherence time of the optical signal $\left(\Delta \quad t_{\text {coh }}\right)$, the Mach-zehnder interferometer is said to operate in the incoherent regime. Otherwise, if $\Delta \mathrm{t}$ tcoh, the interferometer will be in the coherent regime. Specifically, the self-homodyne linewidth measurement technique operates in the incoherent regime. In the incoherent interference regime, optical signals pass through the two interferometer branches and then combine incoherently at the second directional coupler. In this case, the two terms in Equation (2) are not correlated with each other because the differential phase term is not deterministic, which resembles the mixing between lights from two independent laser sources with identical spectral linewidth. Therefore, the normalized power spectral density of the photocurrent in this self-homodyne setup is the auto convolution of the signal power spectral density Sp.s (f) as given by the following equation.

$$
S_{I F}(f)=\operatorname{Sp.s}(\mathrm{f}) \times \operatorname{Sp} . \mathrm{s}(-\mathrm{f})=\frac{1}{1+\left({ }^{f} / \Delta v s\right)^{2}}
$$

Note that here the intermediate frequency (IF) is 0 and the measured RF signal spectral linewidth is twice as wide as the actual optical signal spectral linewidth. Since the center of the RF peak is at zero frequency and the negative part of the spectrum will be flipped onto the positive side, only half the spectrum can be seen on the RF spectrum analyzer. Therefore, the width of this single-sided spectrum is equal to the linewidth of the optical signal [Hui, R. \& O’Sullivan, M. 2008].

\section{System model of Self- homodyne linewidth measurement:}

A linewidth measurement for a commercial tunable laser source (TLS) is proposed. Fig. 2 shows the experimental set-up, which consists of TLS (ANDO AQ4321D), 1 x $23 \mathrm{~dB}$ coupler, a piece of long single mode fiber $(\mathrm{SMF})(25 \mathrm{~km} \& 50 \mathrm{~km})$ length, photo-detector, and $8 \mathrm{GHz}$ radio frequency spectrum analyzer. The input light from TLS is split by the first $3 \mathrm{~dB}$ coupler, which one arm is connected to the second $3 \mathrm{~dB}$ coupler. Another arm of the coupler is connected to a piece of delay SMF connected to the second $3 \mathrm{~dB}$ coupler. The combined light at second coupler is converted into electrical signal by a photodiode, which the signal is analyzed by the radio frequency spectrum analyzer.

Fig. 3 shows the output spectrum of TLS at power setting of $0 \mathrm{dBm}$, linewidth setting of 'wide' and wavelength of $1550 \mathrm{~nm}$. Fig. 4 shows the beat frequency spectrum at three different operating wavelengths. In the experiment, the TLS power and SMF length is fixed at $0 \mathrm{dBm}$ and $25 \mathrm{~km}$, respectively. The linewidths of 120 $\mathrm{MHz}, 110 \mathrm{MHz}$ and $170 \mathrm{MHz}$ are obtained at operating wavelengths of $1530 \mathrm{~nm}, 1540 \mathrm{~nm}$ and $1560 \mathrm{~nm}$, respectively. The values of the linewidth are obtained at $3 \mathrm{~dB}$ from the peak signal from the graphs in Fig. 3.

Fig 5 shows the beat frequency spectrum at three different operating wavelengths but using a longer delay fiber of $50 \mathrm{~km}$. The linewidths of $200 \mathrm{MHz}, 120 \mathrm{MHz}$ and $194 \mathrm{MHz}$ are obtained at operating wavelengths of 1530 $\mathrm{nm}, 1540 \mathrm{~nm}$ and $1560 \mathrm{~nm}$, respectively. It is found that a slightly higher value is obtained with a longer length of the delay fiber.

Fig. 6 shows the linewidth of light against wavelength of the TLS at two different delay fibers. In the experiment, the TLS option is set as 'wide' mode. As shown in the figure, the average linewidth of the TLS is around 200 MHz. Since the result for $25 \mathrm{~km}$ and $50 \mathrm{~km}$ delay fibers are almost similar, it is concluded that $25 \mathrm{~km}$ long of $\mathrm{SMF}$ is enough of provide delay for the self-homodyne linewidth measurement.

\section{Conclusions:}

In this paper, Lasers with very narrow linewidth (high degree of monochromaticity) are proposed, with System model of Self- homodyne linewidth measurement, $25 \mathrm{~km}$ of SMF as a delay fiber, the linewidths of 120 $\mathrm{MHz}, 110 \mathrm{MHz}$ and $170 \mathrm{MHz}$ are obtained at operating wavelengths of $1530 \mathrm{~nm}, 1540 \mathrm{~nm}$ and $1560 \mathrm{~nm}$, respectively. The TLS setting is set at 'wide mode' and the values of the linewidth are obtained at $3 \mathrm{~dB}$ from the peak signal. At $50 \mathrm{~km} \mathrm{SMF}$, the linewidths of $320 \mathrm{MHz}, 120 \mathrm{MHz}$ and $194 \mathrm{MHz}$ are obtained at operating wavelengths of $1530 \mathrm{~nm}, 1540 \mathrm{~nm}$ and $1560 \mathrm{~nm}$, respectively. These results show that a slightly higher value is obtained with a longer length of the delay fiber. The ultra-narrow linewidth measurement is a challenge because the homodyne technique requires long delaying fibers which is impractical for fiber lasers due to propagation losses. 


\section{References}

Alain Kueng, Luc Thevenaz, Philippe A. Robert. (1996). Laser linewidth determination in the sub-Megahertz range using a Brillouin Fibre laser, 22nd European Conference on Optical Communication - ECOC'96, Oslo .

Ch. Spiegelberg, J. Geng, Y. Hu, Y. Kaneda, S. Jiang, and N. Peyghambarian. (2004). Low noise narrow linewidth fiber laser at $1550 \mathrm{~nm}$, J. Lightwave .Technology, vol. 22, no. 1, pp. 57-62, Jan.

Gary L. Wood, Mark A. Dubinskii. (2006). High-power and ultranarrow DFB laser: the effect of linewidth reduction systems on coherence length and interferometer noise" Proc. of SPIE Vol. 6216, 62160C, (2006)·doi: $10.1117 / 12.665675$

Geng J., Staines S., Wang Z., Zong J., Blake, M. \& Jiang S. (2006). Highly stable low-n Brillouin fiber laser with ultra narrow spectral linewidth. IEEE Photon Technol. Lett.18, pp.1813-1815.

Hui, R. \& O’Sullivan, M. (2008). Fiber Optic Measurement Techniques, Elsevier Ltd, Linacre House, Jordan Hill.

K. M. Abramski, W. Rodzen, P. R. Kaczmarek, L. Czurak, A. Budnicki. (2005). Laser Diode Linewidth Measurements, in Proceedings Conference on Lasers and Electro-Optics, pp.: 87-90.

Ludvigsen, Hanne et al. (1998). Laser linewidth measurements using self-homodyne detection with short delay. Optics Communications, Vol. 155, pp. 180-186 Oct.

R. Paschotta. (2010). Derivation of the Schawlow-Townes Linewidth of Lasers. RP Photonics Consulting GmbH R. Phelan R. et al. (2008). Discrete Mode Laser Diodes with Ultra Narrow Linewidth Emission. Eblana Photonics.

Smith, S. P. et al. (1991). Narrow-linewidth stimulated Brillouin fiber laser and applications, Opt.Lett, 16(6).

X. Chen. (2006). Ultra-Narrow Laser Linewidth measurements. PhD thesis, Virginia Tech, 2006.

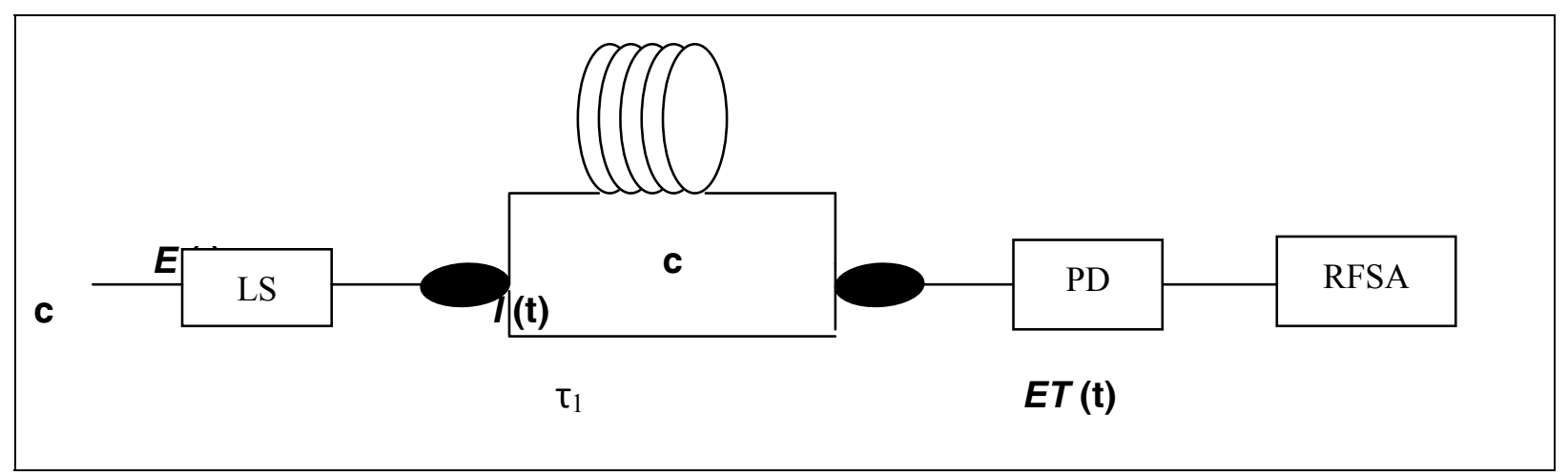

Figure 1. Typical experimental set-up for linewidth measurement using self-homodyne method 


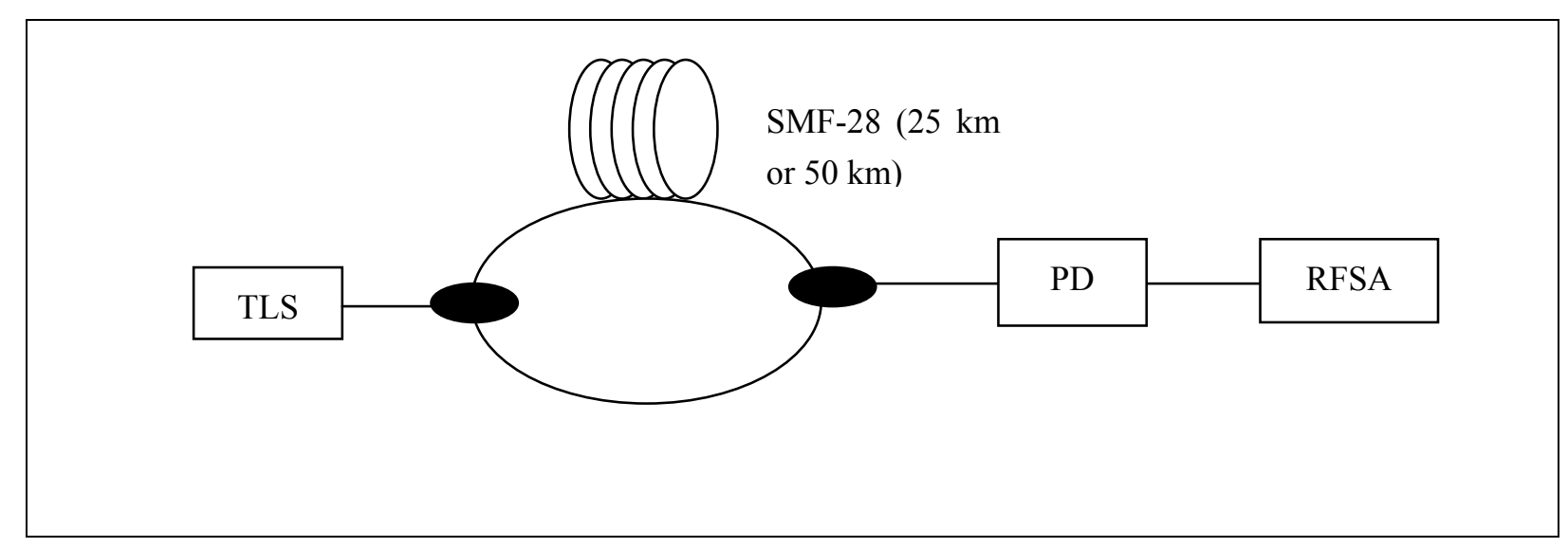

Figure 2. Experimental set-up for self-homodyne linewidth measurement of a TLS

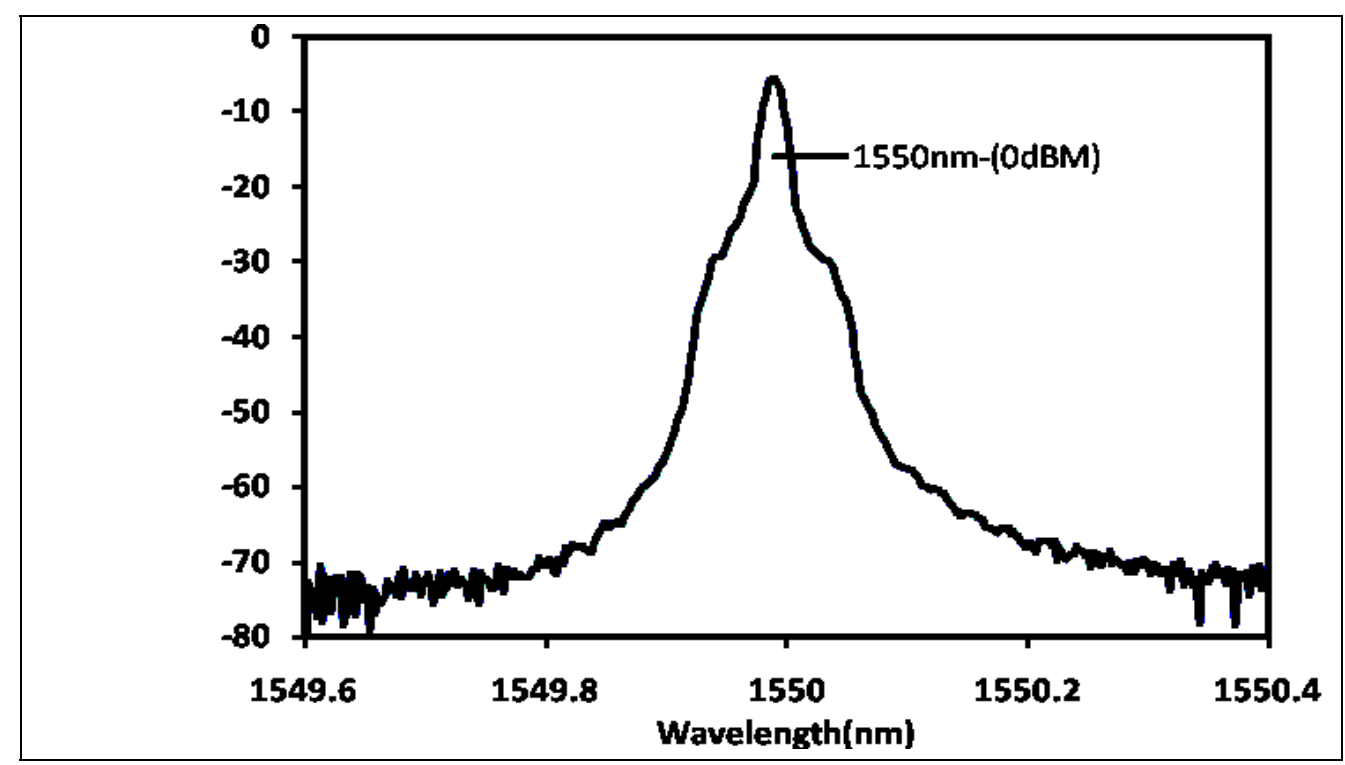

Figure 3. The spectrum of the input signal from TLS 


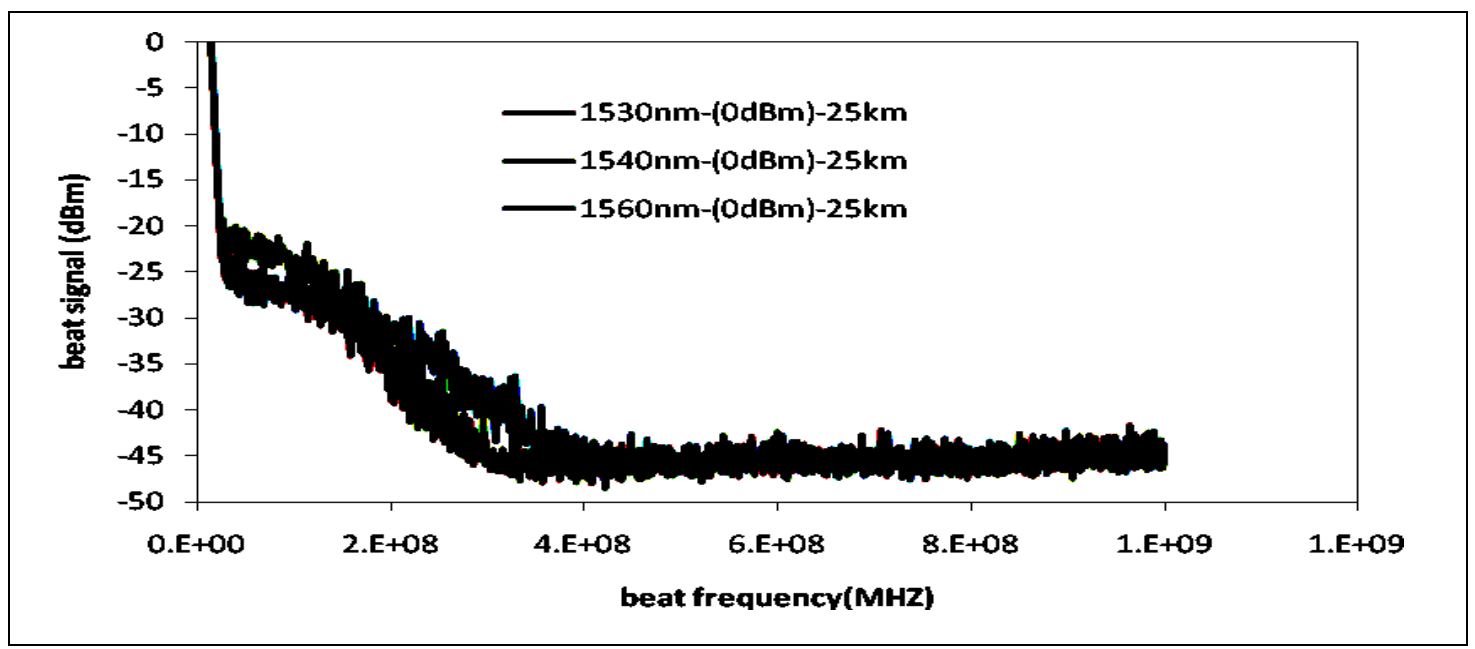

Figure 4 . The beat signal curves against beat frequency at different wavelengths, which were obtained using 25 $\mathrm{km}$ delay fiber

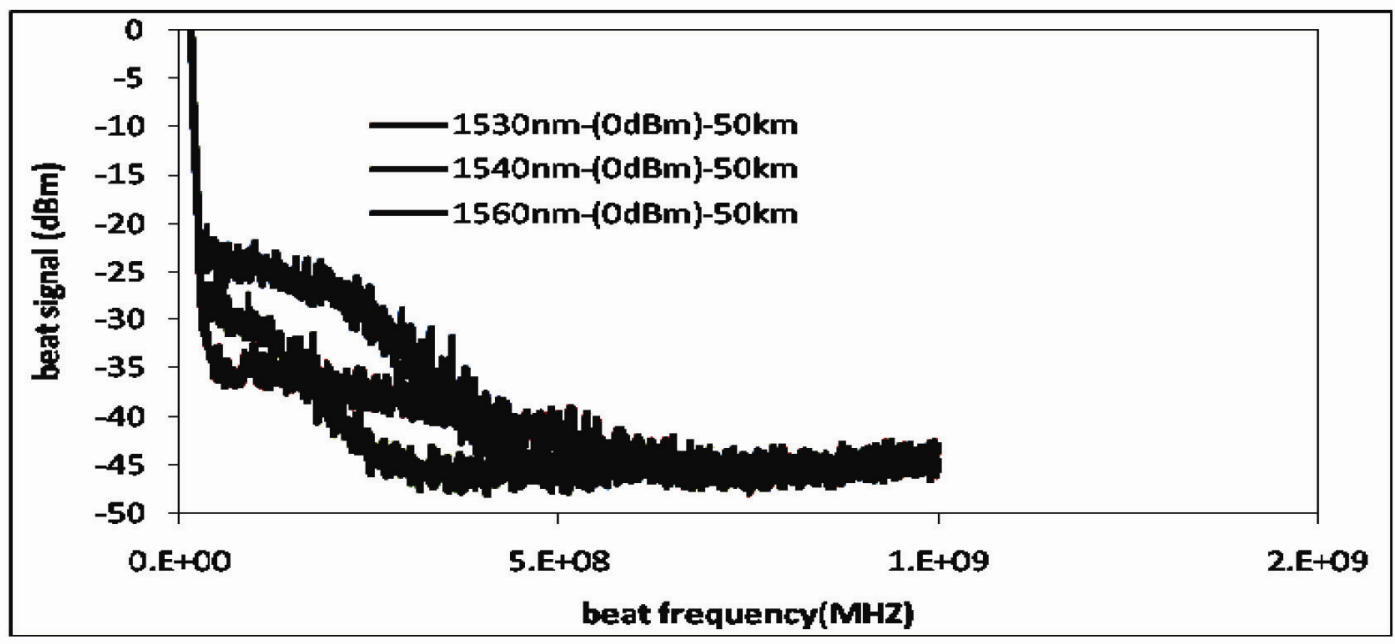

Figure 5. The beat signal curves against beat frequency at different wavelengths, which were obtained using $50 \mathrm{~km}$ delay fiber. 


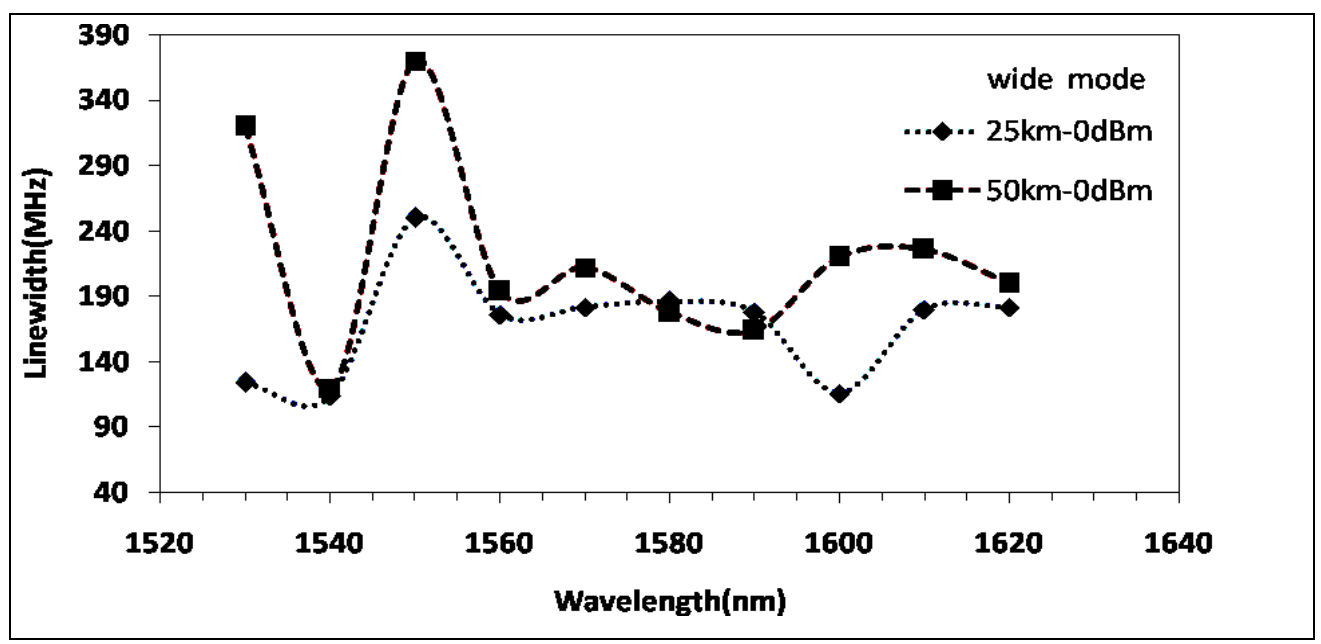

Figure 6. Relationship between linewidth and operating wavelength for the TLS 